\title{
Clearance of Apoptotic Neutrophils Is Diminished in Cord Blood Monocytes and Does Not Lead to Reduced IL-8 Production
}

\author{
CHRISTIAN GILLE, FRANZISKA STEFFEN, KIRSTEN LAUBER, HILDEGARD KEPPELER, ANJA LEIBER, BÄRBEL SPRING, \\ CHRISTIAN F. POETS, AND THORSTEN W. ORLIKOWSKY
}

\begin{abstract}
Department of Neonatology [C.G., F.S., A.L., B.S., C.F.P.], University Children's Hospital, Tuebingen 72076, Germany; Department of Internal Medicine [K.L., H.K.], University Hospital, Tuebingen 72070, Germany; Department of Neonatology [T.W.O.], University
\end{abstract}

Children's Hospital Aachen, Aachen 52074, Germany

\begin{abstract}
Phagocytosis of apoptotic cells, e.g., neutrophils, by monocytes is essential for resolution of inflammation. Delayed removal leads to secondary necrosis, perpetuating inflammation, and tissue destruction. Common histologic features in neonatal chronic inflammatory disorders are an accumulation of apoptotic cells in inflamed tissues. We hypothesized that apoptotic cell removal by monocytes is compromised in newborns. PKH-26 labeled autologous or allogeneic apoptotic neutrophils were fed to monocytes of adult donors (PBMO) and cord blood (CBMO), and phagocytic activity was analyzed by flow cytometry and confocal microscopy. Relative mRNA-expression levels of 21 surface receptors and bridging molecules relevant for apoptotic cell removal were measured, as was postphagocytic IL-8 production upon LPS-stimulation. Compared with PBMO, CBMO exhibited a significantly diminished phagocytotic competence for autologous and allogeneic apoptotic neutrophils. mRNA-expression levels of milk fat globule-EGF factor 8 and T cell immunoglobulin- and mucin-domain-containing molecule, two crucial members of the phagocytic synapse of apoptotic cell removal, were reduced in CBMO. In PBMO, interaction with autologous apoptotic neutrophils reduced LPS-induced IL- 8 production whereas it was enhanced in CBMO. Our data suggest a specific defect in CBMO during clearance of apoptotic neutrophils resulting in impaired anti-inflammatory capacity. (Pediatr Res 66: 507-512, 2009)
\end{abstract}

$\mathrm{P}$ hagocytosis of apoptotic cells by monocytes is a crucial step for normal tissue homeostasis by removing invaded immune cells, e.g., neutrophils, from inflamed tissue $(1,2)$. Delayed removal may lead to secondary necrosis of the apoptotic cell, thus perpetuating inflammation and consecutive tissue destruction. Apart from a safe disposal of apoptotic debris, monocytes secrete anti-inflammatory signals, whereas inflammatory reactions are suppressed, hence enforcing the resolution of inflammation (1). Engulfment is mediated through receptors on the phagocyte, which can either bind structures on the apoptotic cell directly or by the help of bridging molecules, such as mucin-domain-containing molecule E8 (MFG-E8), which form a "phagocytic synapse" (reviewed in Ref. 1).

Received February 4, 2009; accepted July 2, 2009.

Correspondence: Thorsten Orlikowsky, M.D., Department of Neonatology, University Children's Hospital Aachen, Pauwelsstr. 30, 52074 Aachen, Germany; e-mail: torlikowsky@ukaachen.de

Supported by the FORTUNE-program, grant number 1386-0-0, of the Medical Faculty of the University of Tuebingen (C.G.) and Deutsche Forschungsgemeinschaft SFB 685 (K.L.).
During the neonatal period, tissue damage often results in chronic inflammatory responses such as bronchopulmonary dysplasia (BPD), periventricular leucomalacia (PVL), or necrotising enterocolitis (NEC) (3-10). One common histologic feature is the accumulation of apoptotic neutrophils, epithelial, or parenchymal cells in the inflamed tissues $(11,12)$. This observation is unexpected as apoptotic cells are removed rapidly in healthy adults. One reason could be that neonatal monocytes do not engulf apoptotic cells as efficiently as their adult counterparts. Although neonatal monocytes show functional differences compared with those from adults (13), they are not impaired in their phagocytic competence for Grampositive (14) or Gram-negative bacteria in vitro $(15,16)$.

Therefore, we investigated whether neonatal and adult monocytes display different capacities for the internalization of apoptotic neutrophils. To this end, we used a flow cytometric phagocytosis assay using fluorescently labeled neutrophils, which were fed to cord blood monocytes (CBMO) or adult peripheral blood monocytes (PBMO). We also quantified the relative mRNA-expression levels of surface receptors and bridging molecules relevant for apoptotic cell removal and measured the postphagocytic cytokine response in PBMO and CBMO.

\section{MATERIALS AND METHODS}

Patients. The study protocol was approved by the Ethics Committee of Tuebingen University Hospital. Blood was collected from umbilical cords of healthy term neonates immediately after delivery in heparin-coated tubes (4 $\mathrm{IE} / \mathrm{mL}$ blood). These did not exhibit any clinical signs of infection. Mothers with amnion infections and prolonged labor were excluded. All mothers gave written consent before going into labor. Healthy, unrelated adults donated blood and served as controls. ${ }^{1}$

Culture of mononuclear cells. Mononuclear cells from blood of adults and cord (PBMC and CBMC) were isolated by density gradient centrifugation (Biochrom AG, Berlin, Germany) as previously described (17). PBMC and CBMC were resuspended in macrophage-SFM medium (GIBCO, Invitrogen Corporation, Auckland, NZ) with $2 \%$ heat inactivated human $\mathrm{AB}$ serum, counted in an ultra plane Neubauer hemocytometer, placed at $2 \times 10^{6}$ cells $/ \mathrm{mL}$ in flat bottom 96-well cell culture plates (Costar, Bodenheim, Germany) and incubated at $37^{\circ} \mathrm{C}$ with $5 \% \mathrm{CO}_{2}$ for overnight.

\footnotetext{
Abbreviations: CBMC, cord blood mononuclear cells; CBMO, cord blood monocytes; MFG-E8, milk fat globule-EGF factor 8; PBMC, peripheral blood mononuclear cells of adult blood; PBMO, peripheral blood monocytes of adult blood; TIM4, T-cell immunoglobulin- and mucin-domain-containing molecule
} 
Monocyte isolation. For quantitative RT-PCR (qRT-PCR) experiments, monocytes were separated by positive selection using magnetic cell sorting (MACS) CD14 microbeads (Miltenyi Biotec) according to the manufacturer's instructions. The purity of the resulting population was $>95 \% \mathrm{CD} 14$ positive cells as detected by flow cytometry.

Neutrophil purification and labeling. Neutrophils were isolated from the density gradient centrifugation pellet by hypotonic lysis of the remaining erythrocytes using $0.156 \mathrm{M}$ ammonium chloride (18). Neutrophils were washed twice with PBS (Biochrom) and counted with an ultra plane Neubauer hemocytometer. Purity of neutrophils was assessed by SYSMEX-XE2100 hemocytometer as $>95 \%$. Neutrophils were labeled with PKH26 red fluorescent cell linker kit (Sigma Chemical Co., Crailsheim, Germany) according to the manufactuer's instructions. Stained neutrophils were resuspended again in macrophage-SFM medium (GIBCO, Invitrogen Corporation) with $2 \%$ heat inactivated human $\mathrm{AB}$ serum.

Neutrophil apoptosis. To induce apoptosis, neutrophils were serumstarved overnight $(19,20)$. We used only a minimum of macrophage-SFM medium $\left(1 \times 10^{8}\right.$ cells $\left./ \mathrm{mL}\right)$ at $37^{\circ} \mathrm{C}$ with $5 \% \mathrm{CO}_{2}$. Apoptotic neutrophils were identified by Annexin V-FITC staining (BD Biosciences, Heidelberg, Germany; 21). Cells were pelleted $(300 \times \mathrm{g} 5 \mathrm{~min})$ and resuspended in $50 \mu \mathrm{L}$ Annexin-buffer $(0.02 \%$ sodium azide in HEPES-buffer, $\mathrm{pH} 7.4), 10 \mu \mathrm{L}$ Annexin V-FITC was added, for $15 \mathrm{~min}$ at $4^{\circ} \mathrm{C}$ in the dark. PBS $(4 \mathrm{~mL})$ was added, neutrophils were pelleted again, resuspended in PBS, and analyzed. Apoptotic cells were differentiated from necrotic cells by propidium iodide (PI) staining.

Coculture of monocytes with apoptotic neutrophils. Coculture experiments were carried out with PBMC or CBMC from one adult or neonatal donor either with their autologous or with allogeneic apoptotic neutrophils. After $24 \mathrm{~h}$ of preincubation, PBMC and CBMC were cocultured with stained apoptotic autologous (PBMC and CBMC, $n=12$ ) or allogeneic neutrophils (PBMC, $n=12$; CBMC, $n=6$ ) at a ratio of 1:8 (monocytes:neutrophils). After $1,3,6,12$, and $24 \mathrm{~h}$, monocytes were stained with anti-CD14-FITC $\mathrm{MAb}$ (mAB, BD Biosciences), and cells were analyzed on a FACSCalibur flow cytometer (BD Biosciences). Monocytes were gated by size (forward scatter, FSC), granularity (side scatter, SSC), and CD14 expression. The percentage of phagocytosis was defined as percentage of $\mathrm{CD} 14^{+}-\mathrm{PKH} 26^{+}$ events out of $\mathrm{CD} 14^{+}$events. To estimate extracellular binding of apoptotic cells, actin-dependent phagocytosis was blocked by cytochalasin D (CCD; 10 $\mu \mathrm{g} / \mathrm{mL}$; Sigma Chemical Co.) $30 \mathrm{~min}$ before starting addition of apoptotic neutrophils to mononuclear cells.

Real-time qRT-PCR analysis. The measurement of phagocyte receptorand bridging protein mRNA levels was performed by SYBR green real-time qRT-PCR analysis using the ABI Prism 7000 Sequence Detection System (Applied Biosystems, Foster City, CA) and qPCR Mastermix Plus (Eurogentec, Seraing, Belgium). Primers were designed to generate amplicons spanning exon-exon junctions to avoid genomic DNA amplification and were synthesized by MWG Biotech (Ebersberg, Germany). The following primers were used: MFG-E8 forward 5' GCACTCTGCGCTTTGAGCTA 3', MFG-E8 reverse 5' TTGTCAGGG ATGCTGTTATTCTTC 3', T cell immunoglobulin- and mucin-domain-containing molecule (TIM 4) forward $5^{\prime}$ GTGTCAATGTGGAAAACGAGTGATT 3', TIM 4 reverse 5' TGTTCTGCTCAGGAACTGCTGTATC $3^{\prime}$. Total RNA 4 to $9 \times 10^{6}$ CD14-positively selected PBMO or CBMO was extracted according to the manufacturer's instructions with TRIzol Reagent (Invitrogen Life Technologies, Karlsruhe, Germany). Then, $0.5-2 \mu \mathrm{g}$ of total RNA were reversely transcribed with 200 U Superscript RT II reverse transcriptase (Invitrogen Life Technologies) in the presence of $50 \mu \mathrm{M}$ random hexamers (Amersham Biosciences, Freiburg, Germany), $400 \mu \mathrm{M}$ dNTPs (Promega, Heidelberg, Germany), and $1.6 \mathrm{U} / \mu \mathrm{L}$ RNAsIn (Invitrogen Life Technologies) in of a final volume of $25 \mu \mathrm{L}$. Thirty to $60 \mathrm{ng}$ of the resulting cDNA were applied to the following qRT-PCR analyzes (20 $\mu \mathrm{L}$ final volume) with $300 \mathrm{nM}$ primers in $1 \times$ qPCR Mastermix Plus (Eurogentec, Seraing, Belgium) and amplified with the standard temperature profile $\left[2 \mathrm{~min} 50^{\circ} \mathrm{C}, 10 \mathrm{~min} 95^{\circ} \mathrm{C}, 40 \times\left(15 \mathrm{~s} 95^{\circ} \mathrm{C}, 1 \mathrm{~min} 60^{\circ} \mathrm{C}\right)\right]$. Relative quantification was performed using the standard curve method. The results for target gene expression were normalized on 18S rRNA as endogenous control, and the mean values of the PBMO population were set as calibrator.

Confocal microscopy. PBMC were allowed to attach to the glass coverslips overnight at $37^{\circ} \mathrm{C}$ in $5 \% \mathrm{CO}_{2}$. On the consecutive day, autologous apoptotic PKH26-stained neutrophils were added at a ratio of monocytes to neutrophils of $1-8$. After coculturing for $24 \mathrm{~h}$ at $37^{\circ} \mathrm{C}$, nonadherent cells were gently rinsed off with PBS and monocytes were stained for 10 min with anti-CD14-APC mAB (BD Biosciences). The glass coverslips were mounted in Fluoprep-mounting-medium (bioMérieux, Marcy l'Etoile, France) and analyzed with a Leica-DM-IRE2 confocal laser-scanning microscope (Leica; Bensheim, Germany). Fluorescence images were acquired sequentially to avoid nonspecific channel interference. Phagocytosis was confirmed by means of a $z$-axis scanning. Images were digitally processed with Photoshop7.0 (Adobe Systems, Mountain View, CA).

Intracellular cytokine staining. Isolated monocytes were placed at $2 \times$ $10^{6}$ cells $/ \mathrm{mL}$ in flat bottom 24 -well cell culture plates (Costar, Bodenheim, Germany) and incubated at $37^{\circ} \mathrm{C}$ in the presence of brefeldin $(10 \mu \mathrm{g} / \mathrm{mL}$; Sigma Chemical Co.). Apoptotic neutrophils were added in a ratio of 1-8 (monocytes to neutrophils). Thereafter, cells were activated by LPS from Escherichia coli $(1 \mu \mathrm{g} / \mathrm{mL}$; Sigma Chemical Co.). After $20 \mathrm{~h}$ of coculture, cells were resuspended in washing buffer [PBS supplemented with $0.1 \%$ BSA (fraction V; Sigma Chemical Co.) and $0.1 \%$ sodium azide (Sigma Chemical Co.)], centrifuged $\left(300 \times \mathrm{g} 10 \mathrm{~min} 4^{\circ} \mathrm{C}\right)$, fixed and permeabilized by adding $100 \mu \mathrm{L}$ of fix and perm-reagent (BD Biosciences) for $15 \mathrm{~min}$, washed in saponin buffer [PBS supplemented with $0.5 \%$ BSA, 0.1\% saponin (Sigma Chemical Co.), and $0.02 \%$ sodium azide], and stained with anti-IL-8-PE mAB (Diaclone, Starnford, USA) or an isotype control at $4^{\circ} \mathrm{C}$ in the dark for 30 min. Cells were washed twice in saponin buffer and analyzed immediately by flow cytometry. Permeability of cells was assessed by anti-ZAP-70 staining (Caltag/Invitrogen, Burlingame, CA).

Statistical analysis. Fluorescence intensities were determined, and nonspecific background staining was subtracted. Results are expressed as mean \pm SD. Statistical analysis was performed using the $t$ test (Sigmaplot 2000 software for Windows, SPSS, Chicago, IL). Values of $p<0.05$ were considered significant.

\section{RESULTS}

Neutrophil apoptosis and phagocytosis of apoptotic neutrophils by PBMO and CBMO. Assessment of neutrophil apoptosis was performed before each coculture with monocytes, revealing equal proportions of cells from neonates (74 $\pm 20 \%)$ and adults $(83 \pm 11 \%, n=6$, n.s. versus. neonates; Fig. $1 A$, right; one representative experiment is shown in Fig. 1A, left).

PKH26-labeled, autologous neutrophils, which had become apoptotic overnight, were fed to corresponding 1-day-old PBMC and CBMC, respectively. Phagocytosis was analyzed for a period of $24 \mathrm{~h}$. We examined monocyte CD14 expression during the time of cocultivation: Basal expression tended to be lower on CBMC compared with PBMC and was diminished on both upon coculture with neutrophils but still detectable by flow cytometry (Table 1). CD14 expression on neutrophils was not detectable (not shown).

Because phagocytosis was calculated as percentage of $\mathrm{PKH} 26^{+} / \mathrm{CD} 14^{+}$of all $\mathrm{CD} 14^{+}$cells, our data relate to the phagocytic activity from $\mathrm{CD} 14^{+}$cells and termed to be of monocytic origin, although other mononuclear cells were present. Representative dot plots are given in Fig. 1B. After $1 \mathrm{~h}$, phagocytosis of apoptotic cells was equivalent in PBMO $(36 \pm 14 \%)$ and CBMO $(29 \pm 14 \%, n=12, p=0.25$; Fig. $1 B$, right). With prolonged incubation time, a significant difference in the uptake became apparent and persisted during the observation period of $24 \mathrm{~h}$. After $24 \mathrm{~h}, 87 \pm 11 \%$ of PBMO versus $62 \pm 23 \%$ of CBMO had participated in phagocytosis ( $n=12, p=0.018$; Fig. $1 B$, right). The mean PKH26-fluorescence intensities were equivalent, suggesting no difference in the amount of ingested apoptotic prey per monocyte (not shown). Phagocytosis was greatly reduced by preincubation of PBMC with CCD (Fig. $1 B$, right). Intracellular localization was confirmed by confocal laser scanning microscopy (Fig. 1C), showing apoptotic neutrophils (red) in monocytes (green).

In the autologous setting, postphagocytic reactions may depend on the apoptotic prey. In a second cocultivation, we therefore used apoptotic neutrophils, descending from one 

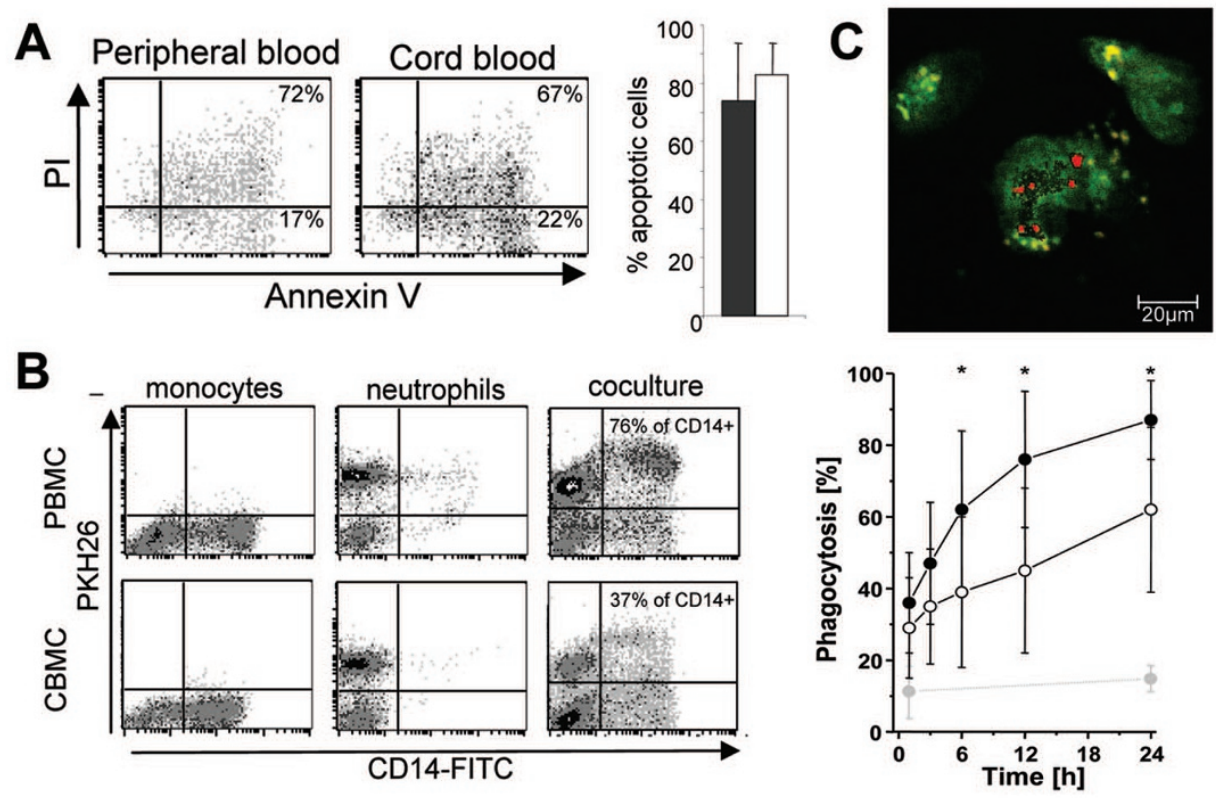

Figure 1. Neutrophil apoptosis $(A)$ and phagocytosis of autologous apoptotic neutrophils by PBMO and CBMO $(B, C)$. Neutrophil apoptosis was assessed after serum starving overnight. Annexin V positive cells were classified as early apoptotic, Annexin V, PI double positive cells as late apoptotic cells. A, left: Representative neutrophil dot plots from adults (left) and cord (right). A, right: Mean and SD of six experiments are depicted (black bar adult, open bar cord). B: Dot plots of anti-CD14-FITC stained PBMC (top left) and CBMC (bottom left), PKH-26 stained neutrophils (middle), and coincubated cells (right) are depicted. Phagocytic activity was calculated as percentage of $\mathrm{PKH}-26^{+} \mathrm{CD} 14^{+}$cells of all CD14 ${ }^{+}$cells. Mean and SD of experiments from 12 different adult and neonatal donors are depicted [right: PBMO, black; CBMO, white; preincubation with cytochalasin $\mathrm{D}$ ( $n=3$ different donors), gray; asterisks depict $p<$ 0.05]. C: Intracellular localization of neutrophils was confirmed by confocal laser scanning microscopy (monocytes, green; neutrophils, red).

Table 1. CD14 expression on monocytes during cocultivation with autologous and allogeneic apoptotic neutrophils

\begin{tabular}{|c|c|c|c|c|c|c|c|c|}
\hline \multirow[b]{2}{*}{ Time (h) } & \multicolumn{4}{|c|}{ PBMC CD14 expression (MFI) } & \multicolumn{4}{|c|}{ CBMC CD14 expression (MFI) } \\
\hline & Control & $\begin{array}{l}\text { Autologous } \\
\text { neutrophils }\end{array}$ & $\begin{array}{l}\text { Allogeneic } \\
\text { adult } \\
\text { neutrophils }\end{array}$ & $\begin{array}{c}\text { Allogeneic } \\
\text { neonatal } \\
\text { neutrophils }\end{array}$ & Control & $\begin{array}{c}\text { Autologous } \\
\text { neutrophils }\end{array}$ & $\begin{array}{l}\text { Allogeneic } \\
\text { adult } \\
\text { neutrophils }\end{array}$ & $\begin{array}{c}\text { Allgeneic } \\
\text { neonatal } \\
\text { neutrophils }\end{array}$ \\
\hline 1 & $221 \pm 75$ & $186 \pm 66$ & $138 \pm 43$ & $116 \pm 66^{*}$ & $156 \pm 46$ & $90 \pm 13^{*}$ & $106 \pm 40^{*}$ & $68 \pm 10^{*}$ \\
\hline 3 & $234 \pm 133$ & $150 \pm 79$ & $121 \pm 53$ & $93 \pm 56$ & $162 \pm 49$ & $101 \pm 31$ & $124 \pm 56$ & $112 \pm 33$ \\
\hline 6 & $231 \pm 166$ & $162 \pm 46$ & $127 \pm 60$ & $116 \pm 44$ & $160 \pm 31$ & $106 \pm 29$ & $120 \pm 39$ & $95 \pm 38$ \\
\hline 12 & $193 \pm 119$ & $112 \pm 46$ & $101 \pm 38$ & $98 \pm 56$ & $172 \pm 17$ & $114 \pm 43$ & $108 \pm 51$ & $85 \pm 36$ \\
\hline 24 & $363 \pm 80$ & $168 \pm 119^{*}$ & $120 \pm 88^{*}$ & $146 \pm 108^{*}$ & $262 \pm 78$ & $161 \pm 53$ & $143 \pm 73$ & $163 \pm 51$ \\
\hline
\end{tabular}

PBMC and CBMC were cocultured with autologous neutrophils and with allogeneic neutrophils of adult and neonatal origin for the times indicated, stained with anti-CD14-FITC, and analyzed by flow cytometry. PBMC and CBMC cultured without neutrophils served as controls $(n=6 ; * p<0.05$ vs. control).

source: Neonatal neutrophils from one cord blood were fed to CBMO (autologous) and to PBMO (allogeneic). Again, CBMO exhibited a significantly reduced internalization of the apoptotic prey cells throughout the observation period. After $24 \mathrm{~h}, 92 \pm 7 \%$ of PBMO versus $66 \pm 25 \%$ of CBMO had ingested apoptotic neutrophils ( $n=6-12, p=0.04$; Fig. $2 A$ ).

In a third approach, we chose a complete allogeneic setup, in which adult apoptotic neutrophils were fed to CBMO and neonatal apoptotic neutrophils were fed to PBMO to avoid possible effects of allogenecity. Notably, the percentage of phagocyting CBMO was higher than in the autologous setting, but still it was significantly lower (at 4 of 5 time points of observation) than the percentage of PBMO: After $24 \mathrm{~h}, 92 \pm$ $7 \%$ of PBMO versus $81 \pm 12 \%$ of CBMO had participated in phagocytosis $(n=6-12, p=0.04$; Fig. $2 B)$. In summary, our results suggest that the CBMO-inherent phagocytic capacity for autologous and allogeneic apoptotic neutrophils is inferior to that of PBMO.
Expression of surface receptors and bridging proteins involved in the recognition of apoptotic cells in PBMO and CBMO. We performed an initial qRT-PCR screen comparing the relative mRNA-expression levels of receptor and bridging protein candidates involved in apoptotic cell recognition in three $\mathrm{CBMO}$ and three PBMO samples. The rationale was to find candidate genes, which in CBMO are less abundantly expressed than in PBMO. In case of two genes encoding for MFG-E8 and TIM4, the mean mRNA-expression level in CBMO was $<50 \%$ of the mean mRNA-expression level in PBMO (data not shown). Therefore, we performed a validation screen, in which only the mRNA-levels of MFG-E8 and TIM4 were measured in a cohort of seven CBMO and six PBMO samples. The second screen confirmed the initial results and again revealed a $>2$-fold reduced mRNA-level of MFG-E8 and TIM4 in CBMO (Fig. 3).

IL-8 production upon ingestion of autologous apoptotic neutrophils. We measured the LPS induced IL-8 production 


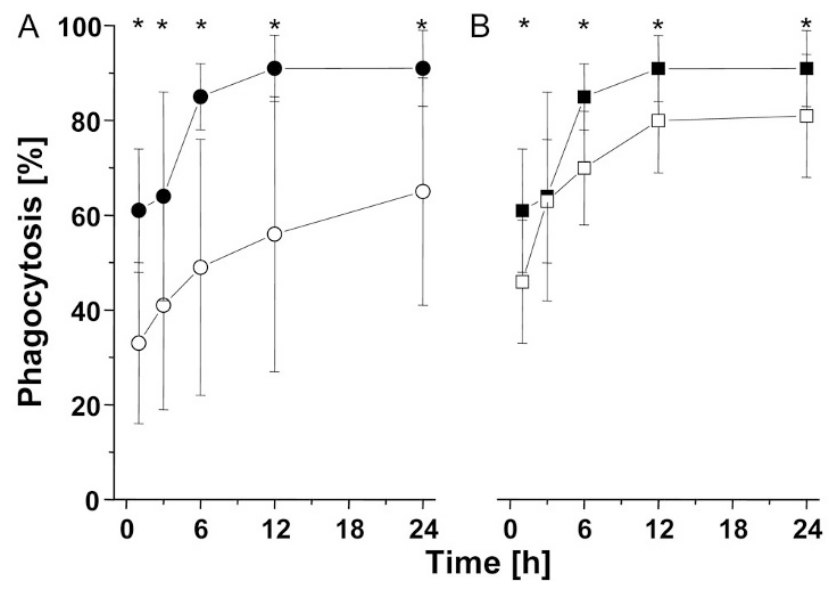

Figure 2. Phagocytosis of autologous and allogeneic apoptotic neutrophils by PBMO and CBMO. A: Phagocytosis of apoptotic neutrophils from six neonatal donors by the corresponding CBMO (autologous, open symbols, $n=$ 6) and 12 neonatal donors by one of 12 different PBMO (allogeneic, $n=12$, black symbols). B: Phagocytosis of apoptotic neutrophils from six different adult by one of six different CBMO (allogeneic, $n=6$, black squares) and 12 different neonatal apoptotic neutrophils by one of 12 different PBMO (allogeneic, $n=12$, open squares). Asterisks depict $p<0.05$.

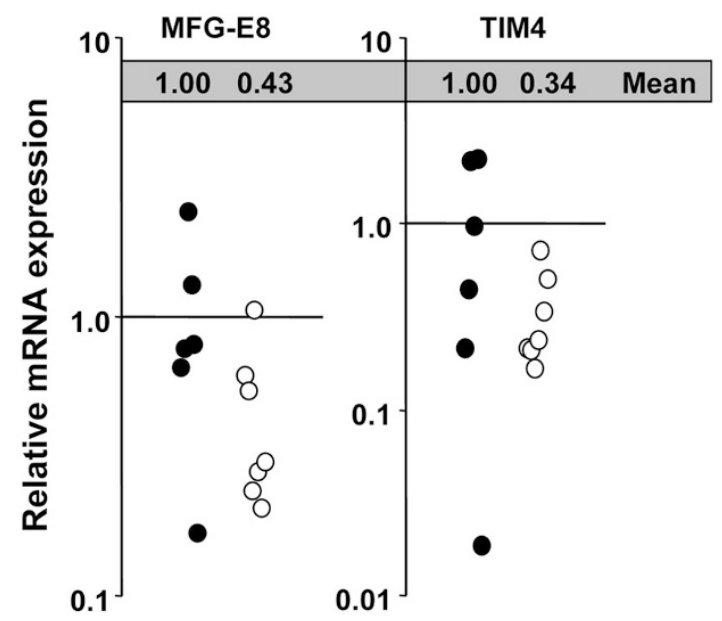

Figure 3. MFG-E8 and TIM4 mRNA-expression levels in PBMO and CBMO. PBMO ( $n=6$, black symbols) and CBMO ( $n=7$, open symbols $)$ were isolated by CD14-positive selection. mRNA-expression levels of MFG-E8 and TIM4 were analyzed by real-time PCR. Values were normalized on 18S rRNA and the mean expression level of PBMO was set as 1 .

in the presence or absence of autologous apoptotic neutrophils. Figure $4 A$ shows representative histograms of IL-8 production by PBMO (upper diagrams) and CBMO (lower diagrams). Monocytes without LPS stimulation did not produce IL-8, measured as percentage of IL-8 producing cells (Fig. $4 B$, left) and mean IL-8 production per cell (Fig. $4 B$, right). LPS induced IL-8 production in PBMO (71 $\pm 21 \mathrm{MFI}$, $n=4)$ and CBMO (72 $\pm 24 \mathrm{MFI}, n=4)$. In the presence of autologous, apoptotic neutrophils, LPS-induced IL-8 production of PBMO was decreased ( $42 \pm 3 \mathrm{MFI}, n=4 ; p=0.04$ $v s$ LPS alone). Contrarily, CBMO responded even with an increase in mean IL-8 production (106 $\pm 17 \mathrm{MFI}, n=4 ; p=$ 0.09 vs LPS alone). Cocultivation with autologous, apoptotic neutrophils alone did not induce IL-8 production. These results suggest that CBMO may display a more proinflammatory postphagocytic response.

\section{DISCUSSION}

In this study, we compared the interaction of neonatal and adult monocytes with autologous and allogeneic apoptotic neutrophils with respect to phagocytosis and postphagocytic cytokine response and analyzed expression of phagocytosisrelevant receptors. In CBMO, we observed (i) a decreased phagocytic activity for autologous and allogeneic apoptotic neutrophils, (ii) a reduced expression of MFG-E8 and TIM4 mRNA, and (iii) no inhibitory effect on IL-8 production upon ingestion of apoptotic neutrophils.

Although previous studies, including our own, described equivalent phagocytic competence of neonatal and adult monocytes for different types of prey, such as Gram-negative bacteria $(15,17)$, Gram-positive (14) bacteria, and latex beads (Ch.G. and Th.O. unpublished observation), we show here that the ingestion of autologous as well as allogeneic apoptotic neutrophils in CBMO is strongly reduced.

A caveat of these results is that the depicted differences in the phagocytic uptake of autologous apoptotic neutrophils could also be due to differences in the apoptosis-rate of the used autologous prey cells. Experimental evidence for this precaution has been presented by others, who demonstrated that in neonatal, neutrophils apoptosis was protracted in vitro $(19,22)$, and results from our own group, showing protracted apoptosis of neonatal monocytes, hinting toward a higher resistance of neonatal hematopoetic cells toward apoptosis. However, we carefully compared the apoptotic populations of neonatal and adult neutrophils by Annexin V-FITC staining and could not detect differences in apoptosis rates, maybe due to special culture conditions: in our experiments, neutrophils were cultivated in a minimum of medium in the absence of serum.

In our autologous/allogeneic setting, phagocytic activity by neonatal monocytes was still impaired although monocytes from adult blood were fed with the same apoptotic neonatal neutrophils (Fig. 2A), and even in a completely allogeneic setting, neonatal monocyte phagocytosis remained inferior compared with their adult counterparts. Thus, we speculate that the uptake of apoptotic neutrophils is specifically impaired in neonatal monocytes. This is in line with former reports of an impaired uptake of lymphoblastoid transformed apoptotic lymphocytes by neonatal monocyte-derived dendritic cells (23) and may hint toward a specific defect in recognition of different apoptotic cell types.

Our results show that the amount of apoptotic neutrophils ingested per monocyte was equivalent, but that a larger percentage of CBMO did not participate in phagocytosis compared with PBMO. Basal CD14 receptor expression, which was shown to be one part of the phagocytic synapse during uptake of apoptotic cells (1), tended to be lower on CBMO compared with PBMO (Table 1). Although this reached no statistical significance, data from other groups showed significantly reduced CD14 expression on neonatal monocytes from preterm infants (24). Therefore, it might be reasonable to speculate that reduced CD14 expression might be in part accounting for diminished phagocytosis of apoptotic neutrophils by CBMO and that phagocytosis of apoptotic cells may 
A

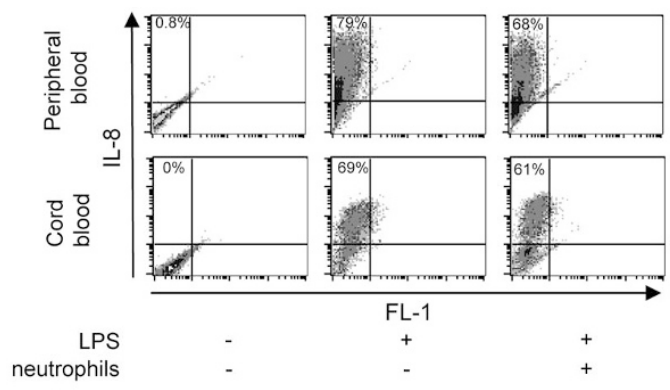

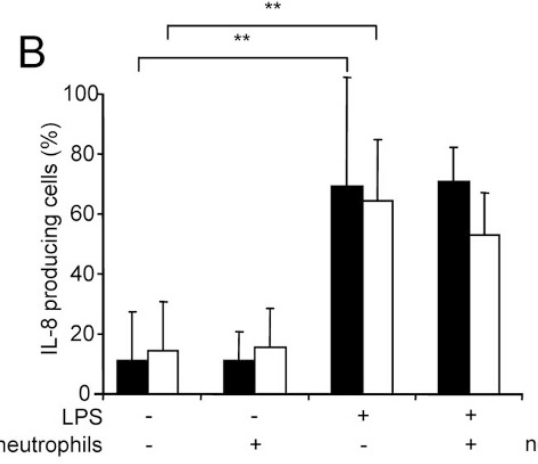

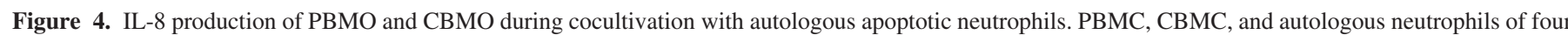

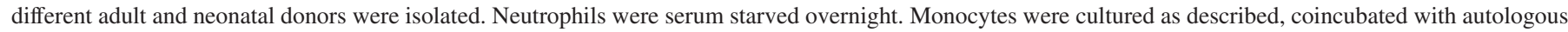

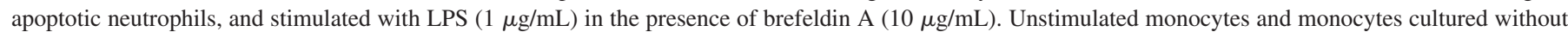

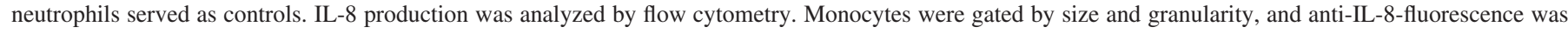

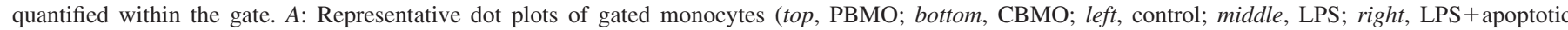

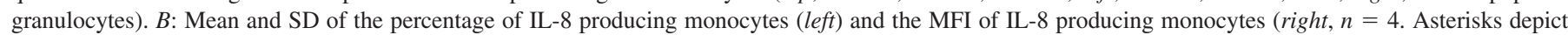
$p<0.05$ and double asterisks depict $p<0.005$ ).

be an acquired function of monocytes upon differentiation. This is supported by Miller et al. (25), who described a reduced phagocytic activity of naïve blood monocytes compared with differentiated peritoneal macrophages and correlated this to the increased intracellular expression of 12/15 lipoxygenase during differentiation. With this in mind, it might be interesting to investigate monocyte functions of preterm infants to analyze whether phagocytic function is related to gestational age as well as the function of more differentiated monocyte-derived macrophages. Moreover, it would be interesting to compare the phagocytic ability of adult and neonatal monocytes under inflammatory conditions, because it was shown that neonatal monocytes significantly enhanced certain immune functions such as TLR4 expression (26) and cytokine release (27). Further studies on monocytes functions of neonates are necessary.

Apart from 12/15 lipoxygenase, other factors are likely to contribute to the phagocytic activity. Because previous studies could not detect significant differences between neonatal and adult monocytes with respect to bacteria or microsphere phagocytosis $[(15,17)$; C.G. and T.O., unpublished observation], it is feasible that CBMO might have defects in the recognition of apoptotic cells rather than in the process of phagocytosis itself. Therefore, we compared the mRNA expression profile of known members of the phagocytic synapse of apoptotic cell removal in CBMO and PBMO. We observed that two of 21 genes tested displayed a reduced expression in CBMO: MFG-E8 and TIM4, intriguingly crucially involved in the recognition of externalized phosphatidylserine (28-30), the central "eat-me" signal of apoptotic cells (1). This characteristic confines the phagocytic function of MFG-E8 and TIM4 to the recognition of apoptotic cells and represents a fundamental difference to other receptors or bridging proteins, such as CD14, the scavenger receptors, or complement proteins, which also participate in the engulfment of bacteria. Hence, MFG-E8 and TIM4 represent promising candidates, whose reduced expression might be causative for the decreased uptake of apoptotic cells by CBMO. However, further studies are required to validate our mRNA-based observations.

The clearance of apoptotic cells is crucial for the resolution of inflammation, because the safe disposal of apoptotic cells prevents the perpetuation of inflammation by secondary necrosis. Furthermore, after having ingested apoptotic cells, professional phagocytes are known to actively shift their repertoire of secreted cytokines toward anti-inflammation. Thus, it has been shown that TNF-alpha and IL-8 production are inhibited, whereas IL-10 and TGF-beta production could be inhibited or induced (31,32). We showed here that LPSinduced amount of IL-8 produced by PBMO was diminished upon interaction with autologous apoptotic neutrophils, whereas IL-8 production by CBMO was enhanced instead (Fig. 4, right). Although this was true regarding the amount of IL-8 produced, the percentage PBMO and CBMO of IL-8 producing upon LPS stimulation was not altered in the presence of apoptotic neutrophils (Fig. 4, left). We therefore think that the inhibitory effect of apoptotic neutrophils does not lead to a complete silencing of IL-8 production in monocytes but strongly reduce it.

Concerning the biologic relevance, we interpret our in vitro data with caution: our data concentrate on one cell type of the mononuclear phagocyte system, i.e., on blood-derived monocytes. Defects of these cells might be compensated by more differentiated monocyte-derived macrophages located in different tissues. Moreover, the often observed relative monocytosis in newborns may compensate for a partial dysfunction of blood monocytes.

Taken together, our in vitro results suggest that the clearance of apoptotic neutrophils by neonatal monocytes is impaired specifically and does not display the anti-inflammatory character observed in adult monocytes. Whether the impaired recognition of apoptotic cells by neonatal monocytes is also relevant for removal of other apoptotic tissue cells (epithelial cells, parenchymal cells) has to be evaluated. In this regard, it is tempting to speculate that the accumulation of apoptotic cells in inflamed tissues, which frequently can be observed in 
typical neonatal disorders such as BPD, PVL, and NEC, might in part be caused by a defective disposal by monocytes and monocyte-derived cells.

Acknowledgments. We thank Andrea Schäfer, Department of Medical Microbiology, for help with confocal microscopy and Derek Stebbens for carefully reading the manuscript.

\section{REFERENCES}

1. Lauber K, Blumenthal SG, Waibel M, Wesselborg S 2004 Clearance of apoptotic cells: getting rid of the corpses. Mol Cell 14:277-287

2. Savill J, Dransfield I, Gregory C, Haslett C 2002 A blast from the past: clearance of apoptotic cells regulates immune responses. Nat Rev Immunol 2:965-975

3. Bracci R, Buonocore G 2003 Chorioamnionitis: a risk factor for fetal and neonatal morbidity. Biol Neonate 83:85-96

4. Cornette L 2004 Fetal and neonatal inflammatory response and adverse outcome. Semin Fetal Neonatal Med 9:459-470

5. Cornette L 2005 Perinatal inflammation and infection. Minerva Ginecol 57:411-421

6. Hentschel J, Berger TM, Tschopp A, Muller M, Adams M, Bucher HU 2005 Population-based study of bronchopulmonary dysplasia in very low birth weight infants in Switzerland. Eur J Pediatr 164:292-297

7. Holcroft CJ, Blakemore KJ, Allen M, Graham EM 2003 Association of prematurity and neonatal infection with neurologic morbidity in very low birth weight infants. Obstet Gynecol 101:1249-1253

8. Kohelet D, Shochat R, Lusky A, Reichman B 2006 Risk factors for seizures in very low birthweight infants with periventricular leukomalacia. J Child Neurol 21:965970

9. Tapia JL, Agost D, Alegria A, Standen J, Escobar M, Grandi C, Musante G, Zegarra J, Estay A, Ramirez R 2006 Bronchopulmonary dysplasia: incidence, risk factors and resource utilization in a population of South American very low birth weight infants. J Pediatr (Rio J) 82:15-20

10. Vigneswaran R 2000 Infection and preterm birth: evidence of a common causal relationship with bronchopulmonary dysplasia and cerebral palsy. J Paediatr Child Health 36:293-296

11. Das KC, Ravi D, Holland W 2004 Increased apoptosis and expression of p21 and p53 in premature infant baboon model of bronchopulmonary dysplasia. Antioxid Redox Signal 6:109-116

12. Speer CP 2003 Inflammation and bronchopulmonary dysplasia. Semin Neonatol 8:29-38

13. Gille C, Orlikowsky TW 2007 Flow cytometric methods in the detection of neonatal infection. Transfus Med Hemother 34:157-163

14. Gille C, Leiber A, Mundle I, Spring B, Abele H, Spellerberg B, Hartmann H, Poets ChF, Orlikowsky TW 2009 Phagocytosis and postphagocytic reaction of cord blood and adult blood monocyte after infection with green fluorescent protein-labeled Escherichia coli and group B Streptococci. Cytometry B Clin Cytom 76B:271-284

15. Gille C, Spring B, Tewes L, Poets CF, Orlikowsky T 2006 A new method to quantify phagocytosis and intracellular degradation using green fluorescent protein-labeled
Escherichia coli: comparison of cord blood macrophages and peripheral blood macrophages of healthy adults. Cytometry A 69:152-154

16. Gille C, Spring B, Bernhard W, Gebhard C, Basile D, Lauber K, Poets CF, Orlikowsky TW 2007 Differential effect of surfactant and its saturated phosphatidylcholines on human blood macrophages. J Lipid Res 48:307-317

17. Gille C, Leiber A, Spring B, Kempf VA, Loeffler J, Poets CF, Orlikowsky TW 2008 Diminished phagocytosis-induced cell death (PICD) in neonatal monocytes upon infection with Escherichia coli. Pediatr Res 63:33-38

18. Gerber CE, Bruchelt G, Falk UB, Kimpfler A, Hauschild O, Kuci S, Bachi T, Niethammer D, Schubert R 2001 Reconstitution of bactericidal activity in chronic granulomatous disease cells by glucose-oxidase-containing liposomes. Blood 98:3097-3105

19. Hanna N, Vasquez P, Pham P, Heck DE, Laskin JD, Laskin DL, Weinberger B 2005 Mechanisms underlying reduced apoptosis in neonatal neutrophils. Pediatr Res 57:56-62

20. Koenig JM, Stegner JJ, Schmeck AC, Saxonhouse MA, Kenigsberg LE 2005 Neonatal neutrophils with prolonged survival exhibit enhanced inflammatory and cytotoxic responsiveness. Pediatr Res 57:424-429

21. Brown SB, Savill J 1999 Phagocytosis triggers macrophage release of Fas ligand and induces apoptosis of bystander leukocytes. J Immunol 162:480-485

22. Allgaier B, Shi M, Luo D, Koenig JM 1998 Spontaneous and Fas-mediated apoptosis are diminished in umbilical cord blood neutrophils compared with adult neutrophils. J Leukoc Biol 64:331-336

23. Wong OH, Huang FP, Chiang AK 2005 Differential responses of cord and adult blood-derived dendritic cells to dying cells. Immunology 116:13-20

24. Gengenbacher D, Salm H, Vogt A, Schneider H 1998 Detection of cell surface determinants for anti-Leu M3 (CD14), MY9 (CD33) and MY4 (CD14) and phagocytic function of cord blood monocytes in the course of gestational age. Bone Marrow Transplant 22:S48-S51

25. Miller YI, Chang MK, Funk CD, Feramisco JR, Witztum JL 2001 12/15lipoxygenase translocation enhances site-specific actin polymerization in macrophages phagocytosing apoptotic cells. J Biol Chem 276:19431-19439

26. Yerkovich ST, Wikström ME, Suriyaarachchi D, Prescott SL, Upham JW, Holt PG 2007 Postnatal development of monocyte cytokine responses to bacterial lipopolysaccharide. Pediatr Res 62:547-552

27. Hanayama R, Tanaka M, Miwa K, Shinohara A, Iwamatsu A, Nagata S 2002 Identification of a factor that links apoptotic cells to phagocytes. Nature 417:182187

28. Schultz C, Rott C, Temming P, Schlenke P, Möller JC, Bucsky P 2002 Enhanced interleukin-6 and interleukin-8 synthesis in term and preterm infants. Pediatr Res $51: 317-322$

29. Kobayashi N, Karisola P, Pena-Cruz V, Dorfman DM, Jinushi M, Umetsu SE, Butte MJ, Nagumo H, Chernova I, Zhu B, Sharpe AH, Ito S, Dranoff G, Kaplan GG, Casasnovas JM, Umetsu DT, Dekruyff RH, Freeman GJ 2007 TIM-1 and TIM-4 glycoproteins bind phosphatidylserine and mediate uptake of apoptotic cells. Immunity 27:927-940

30. Miyanishi M, Tada K, Koike M, Uchiyama Y, Kitamura T, Nagata S 2007 Identification of Tim4 as a phosphatidylserine receptor. Nature 450:435-439

31. Fadok VA, Bratton DL, Konowal A, Freed PW, Westcott JY, Henson PM 1998 Macrophages that have ingested apoptotic cells in vitro inhibit proinflammatory cytokine production through autocrine/paracrine mechanisms involving TGF-beta, PGE2, and PAF. J Clin Invest 101:890-898

32. Voll RE, Herrmann M, Roth EA, Stach C, Kalden JR, Girkontaite I 1997 Immunosuppressive effects of apoptotic cells. Nature 390:350-351 